\title{
Biermer's disease revealed by extremities hyperpigmentation in a black skin
}

\section{Julienne Noudé Teclessou1, Essohana Padaro², Séfako Akakpo', Laconi Kaaga ${ }^{3}$, Panawe Kassang', Bayaki Saka'}

${ }^{1}$ Department of Dermatology and Venereology CHU Lome, University of Lome, Togo, ${ }^{2}$ Hematology Department, Campus Teaching Hospital, University of Lome, Togo, ${ }^{3}$ Internal medicine department, Campus Teaching Hospital, University of Lome, Togo

Corresponding author: Julienne Noudé Teclessou, MD, E-mail: tjulie06@yahoo.fr

\begin{abstract}
Biermer's disease is an autoimmune disease due to deficiency of vitamin B12 (cyanocobalamin) result of its malabsorption by lack of intrinsic factor. We have reported Biermer's disease case revealed by skin signs. A 47-year-old man consulted in dermatology for hyperpigmentation extremities evolving for 7 years ago. The patient was diabetic for 11 years ago and had ageusia 5 years earlier. The examination noted good general condition and especially hyperpigmentation of his whole hands and feets. The hemoglobin level was $11.2 \mathrm{~g} / \mathrm{dl}$ with an average blood volume of $100.1 \mathrm{fl}$. The diagnosis of Biermer's disease has been confirmed by deficiency of Vitamin B12 (cyanocobalamin) which rate was $0.01 \mathrm{pg} / \mathrm{ml}$ $($ Normal $=208-963.5 \mathrm{pg} / \mathrm{ml})$. The intrinsic antibody factor test was positive. Therefore, the patient received injections of vitamin B12 with disappearance of the skin lesions over the follow up. Our observation suggests that hyperpigmentation of the extremities could be earlier sign of Biermer's disease even on black skin.
\end{abstract}

Key words: Biermer's disease; Hyperpigmentation of the Extremities; Black skin

How to cite this article: Teclessou JN, Padaro E, Akakpo S, Kaaga L, Kassang P, Saka B. Biermer's disease revealed by extremitieBiermer's extremities hyperpigmentation in a black skin. Our Dermatol Online. 2021;12(Supp. 2):26-29.

Submission: 20.03.2021; Acceptance: 17.05.2021

DOI: 10.7241 /ourd.2022s2.6 


\section{Hyperpigmentation des extrémités révélatrice de la maladie de Biermer chez un sujet de peau noire}

\section{Julienne Noudé Teclessou', Essohana Padaro², Séfako Akakpo', Laconi Kaaga ${ }^{3}$, Panawe Kassang', Bayaki Saka ${ }^{1}$}

${ }^{1}$ Department of Dermatology and Venereology CHU Lome, University of Lome, Togo, ${ }^{2}$ Hematology Department, Campus Teaching Hospital, University of Lome, Togo, ${ }^{3}$ Internal medicine department, Campus Teaching Hospital, University of Lome, Togo.

Corresponding author: Julienne Teclessou, MD, E-mail: tjulie06@yahoo.fr

\section{RÉSUMÉ}

La maladie de Biermer est une maladie auto-immune due à une carence en vitamine B12 (cyanocobalamine) par malabsorption; suite à un déficit en facteur intrinsèque. Nous rapportons un cas de maladie de Biermer avec au premier plan des lésions cutanées. Un homme âgé de 47 ans était admis en consultation dermatologique pour une hyperpigmentation des extrémités évoluant depuis 7 ans. Le patient était diabétique type 2 depuis 11 ans et avait présenté 5 ans plus tôt une agueusie. A l'examen, le patient était en bon état général. On notait une hyperpigmentation du dos des mains et des pieds, des paumes et plantes. Le taux d'hémoglobine était à $11,2 \mathrm{~g} / \mathrm{dl}$ avec un volume globulaire moyen de 100,1 fl. La carence en vitamine Bl2 (cyanocobalamine) était confirmée par une cyanocobalémie effondrée à $0,01 \mathrm{pg} / \mathrm{ml}(\mathrm{N}=208-963,5 \mathrm{pg} / \mathrm{ml})$. Le dosage des anticorps anti facteur intrinsèque était positif. Le diagnostic de maladie de Biermer a été retenu et le patient mis sous vitamine B12 injectable. Lévolution a été marquée par une régression des lésions cutanées. Notre observation suggère que l'hyperpigmentation des extrémités pourrait être un signe révélateur de la maladie de Biermer chez le noir africain. Il s’agit de lésion facilement accessible au dermatologiste pour un diagnostic précoce de cette affection sur peau noire.

Mots clés : Maladie de Biermer; Hyperpigmentation des Extrémités; Sujet Noir

\section{INTRODUCTION}

La maladie de Biermer est une maladie auto-immune due à une carence en vitamine $\mathrm{B}$ par malabsorption suite à déficit en facteur intrinsèque [1]. C'est une maladie ubiquitaire dont la prévalence varie d'une région à une autre. En Afrique, des cas ont été rapportés au Zimbabwe, Nigéria, Sénégal, Togo, Burkina-Faso ; mais la prévalence réelle n’est pas connue [2-6]. La maladie de Biermer se manifeste généralement par des signes cliniques d'une anémie (pâleur conjonctivale), des atteintes neurologiques à type de paresthésies ou de troubles profonds de la sensibilité [6,7], une atteinte médullaire [6] ou encore des signes digestifs notamment la glossite de Hunter [5]. Il est aussi possible d'observer des atteintes cutanéo-muqueuses variées comme des hyperpigmentations muqueuses, voir des signes d'une mélanodermie acquise [8,9]. Nous rapportons un cas d'hyperpigmentation des extrémités révélatrice d'une maladie de Biermer chez un sujet de peau noire.

\section{CASE REPORT}

Un patient de 47 ans a été vu en dermatologie, pour une hyperpigmentation des extrémités évoluant depuis 7 ans. Il était vu cinq ans plus tôt en neurologie pour une agueusie associée à picotement de la muqueuse buccale. Le scanner cérébral réalisé à l'époque était normal et le patient était traité sans succès. Dans ses antécédents, le patient était diabétique type 2 depuis 11 ans sous antidiabétique orale (metformine 500). Son père était également diabétique depuis 2 ans sous traitement.

A l'examen, le patient était en bon état général et les conjonctives étaient bien colorées sans ictère. La température était à $37,2^{\circ} \mathrm{C}$ et la tension artérielle

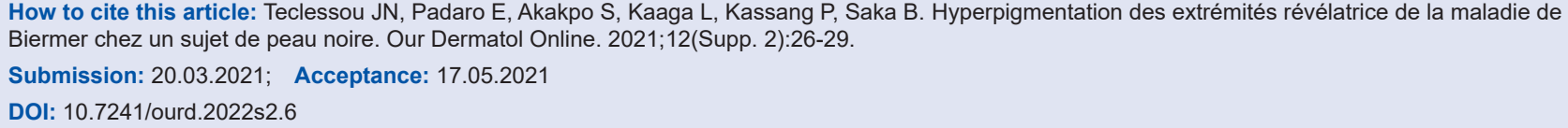


à 125/86 mmHg. Le poids était de $74 \mathrm{Kg}$ pour une taille de $1,7 \mathrm{~m}$, ce qui correspondait à un indice de masse corporelle de $25,6 \mathrm{Kg} / \mathrm{m}^{2}$. On notait au dos des mains et des pieds, aux paumes et plantes, une hyperpigmentation en placard mal limité non squameuse (Fig. la et $1 \mathrm{~b}$ ). La langue était dépapillée lisse avec des macules hyperpigmentées par endroit (Fig. 2). Lexamen du reste du tégument, de la muqueuse génitale et des phanères était normal.

L'hémogramme notait une anémie macrocytaire normochrome avec un taux d'hémoglobine à $11,2 \mathrm{~g} / \mathrm{dl}$ $(\mathrm{N}: 12$ à $16 \mathrm{~g} / \mathrm{dl})$ et un volume globulaire moyen (VGM) de 100,1 fl (normal 82 à $95 \mathrm{fl}$ ); une thrombopénie à 127000 plaquettes $/ \mathrm{mm} 3$. La glycémie à jeun était à $1,68 \mathrm{~g} / 1$ alors que l'urémie et la créatinémie étaient normales. La sérologie VIH était négative. La C-réactive protéine était négative. Le dosage de la vitamine B12 (cyanocobalamine) était à $0,0 \mathrm{lpg} / \mathrm{ml}(\mathrm{N}=208$ $963,5 \mathrm{pg} / \mathrm{ml})$. Le dosage des anticorps anti facteur intrinsèque était positif. La fibroscopie oesogastroduodénale avec biopsie n'a pas été faite pour des raisons économiques.

Nous avons donc retenu le diagnostic de maladie de Biermer. Le patient a été mise sous vitamine B12 injectable $1000 \mu \mathrm{g} / 4 \mathrm{ml}$ à raison d'une ampoule par jour pendant 10 jours puis 1 ampoule par mois à vie. L'évolution a été marquée par une régression des lésions cutanées 2 mois après le début du traitement (Fig. 3) et une régression de l'agueusie.

\section{DISCUSSION}

Le diagnostic évoqué devant la présence d'une agueusie, d'une hyperpigmentation des extrémités et des macules hyperpigmentées de la langue était celui d'une maladie de Biermer, qui a été confirmée par une cyanocobalémie effondrée et la présence d'anticorps anti facteur intrinsèque. Cette hyperpigmentation n'a pas été rattachée à son diabète qui évoluant depuis 11 ans pour la simple raison qu'elle n'avait pas un caractère sale pouvant rentrer dans le cadre d'un acanthosis nigricans. La glossite de Hunter, signe clinique caractéristique de la maladie de Biermer était présente chez notre patient 5 ans avant le diagnostic de sa maladie. Cette glossite était associée à une hyperpigmentation de la muqueuse buccale. La présence de macules hyperpigmentées de la langue dans notre observation pourrait rentrer dans le cadre des hyperpigmentations de la muqueuse buccale bien connues au cours de la maladie de Biermer $[8,9]$.
Mais la particularité de notre observation réside dans le fait que l'hyperpigmentation des extrémités (mains, pieds) était la circonstance révélatrice de la maladie de Biermer chez ce patient.

L'hyperpigmentation des extrémités semble être un signe clinique caractéristique de la maladie de Biermer chez le noire Africain. Berthe et al. au Sénégal avaient rapportés la présence de mélanodermie des mains/ pieds ou une hyperpigmentation de la langue chez $100 \%$ des patients atteints de maladie de Biermer [8]. Aussi, toujours au Sénégal, 78,57\% des patients dans l'étude de Diop et al. présentaient également une mélanodermie [9]. Notre observation suggère donc que l'hyperpigmentation des extrémités pourrait être un signe révélateur de la maladie de Biermer, facilement accessible au dermatologiste pour un diagnostic précoce de cette affection sur peau noire.

La maladie de Biermer est souvent rapportée comme associée à une maladie auto-immune notamment une thyroïdite, une beta thalassémie, la cirrhose autoimmune, le vitiligo, le diabète [2,8-11]. Le diabète type 1 a été souvent rapporté comme associé à la

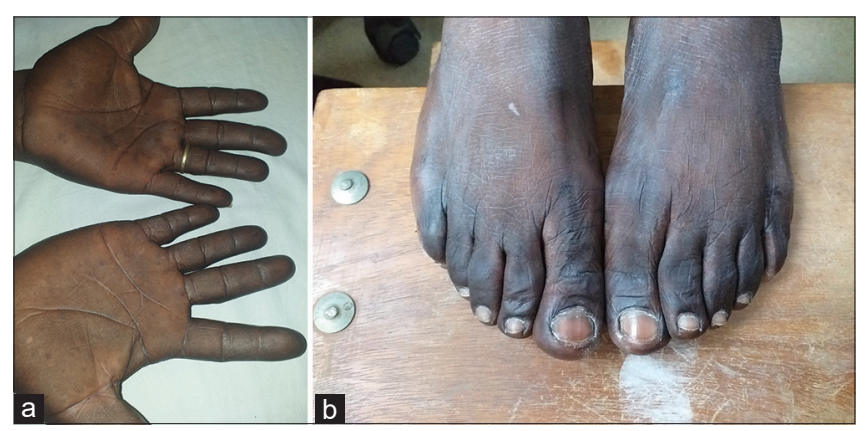

Figure 1 : a) Hyperpigmentation des paumes des mains b) Hyperpigmentation du dos des pieds

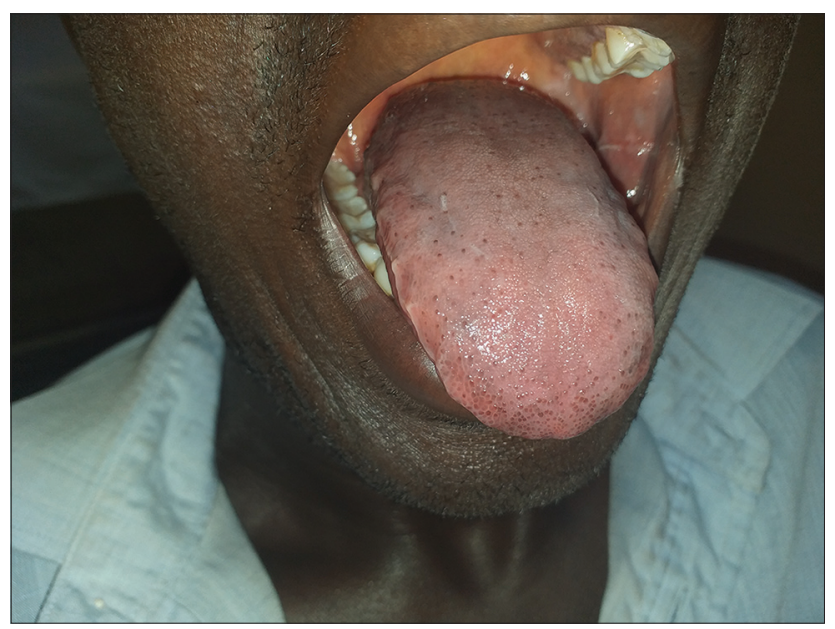

Figure 2 : Glossite de Hunter associée à une hyperpigmentation de la langue 


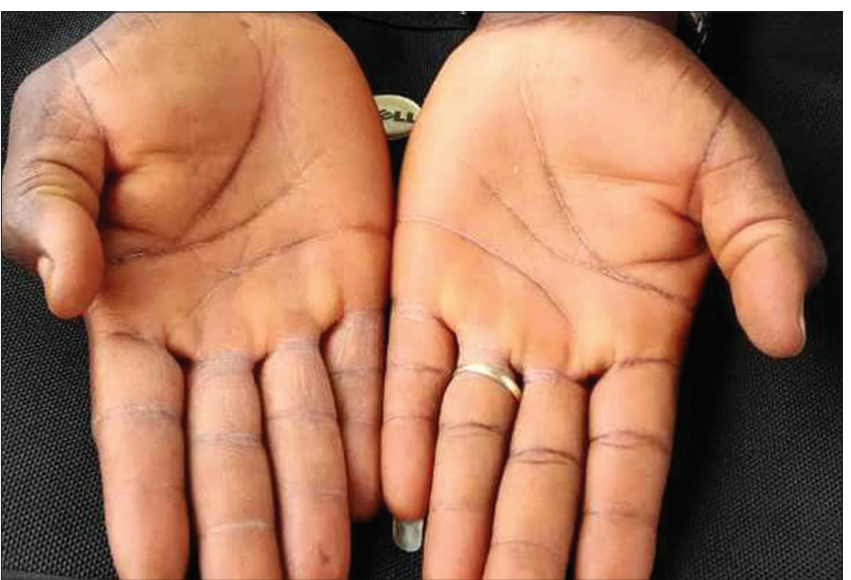

Figure 3 : Régression de l'hyperpigmentation des paumes des mains

maladie [12] Dans notre cas, le patient était diabétique type 2 depuis 11 ans.

Lanémie constitue souvent le signe inaugural de la maladie suivi des troubles neurologique. En effet, la maladie de Biermer se caractérise par une anémie pernicieuse avec un taux d'hémoglobine allant de $1,7 \mathrm{~g} / \mathrm{dl}$ à $10,2 \mathrm{~g} / \mathrm{dl}$ selon différents auteurs $[2,3,6,8,10,12]$. Il s'agit d'une anémie macrocytaire avec un VGM allant de 100 à $132 \mathrm{fl}[2,5,6]$. Cependant, un cas de maladie de Biermer a été rapporté au Sénégal [13] avec un taux d'hémoglobine à 15,2g/dl. Bien que les signes cliniques d'anémie étaient absents chez notre patient, on notait un début d'effondrement du taux d'hémoglobine $(11,2 \mathrm{~g} / \mathrm{dl})$ associé à une macrocytose (VGM à 100,1fl).

Lévolution était marquée par une régression quasitotale de l'hyperpigmentation des extrémités chez notre patient suite à l'administration de la vitamine B12. Lefficacité de la vitamine B12 est bien connu dans la prise en charge de la maladie de Biermer avec généralement une normalisation de l'hémogramme ; une régression de la glossite de Hunter et des signes neurologiques $[6-8,10]$. En dehors de la correction des troubles hématologiques chez notre patient, la régression des signes cutanées observés nous permet de conclure à l'efficacité de la vitamine B12 tant sur l'anémie que sur les signes cutanés de la maladie particulièrement observés chez le sujet noir.

\section{CONCLUSION}

La maladie de Biermer est une affection hématologique bien connue de par l'anémie pernicieuse qu'elle engendre. Cependant, des signes cutanés peuvent être au premier plan chez le sujet noir, permettant de faire un diagnostic précoce.

\section{Consent}

The examination of the patient was conducted according to the principles of the Declaration of Helsinki.

The authors certify that they have obtained all appropriate patient consent forms, in which the patients gave their consent for images and other clinical information to be included in the journal. The patients understand that their names and initials will not be published and due effort will be made to conceal their identity, but that anonymity cannot be guaranteed.

\section{REFERENCES}

1. Binet C, Zandecki M. Hématologie. Société française d'hématologie, Issyles- Moulineaux, Elsevier Masson SAS, 2011, 120-5.

2. Segbena AY, Ambofo-Planche Y, Gbadoe AD, Dogba AD, Kueviakoe IM, Vovor A, et al. [Four case reports of Biermer's anemia in West Africa]. Med Trop. 2003;63:593-6.

3. Padaro E, Kuéviakoé IMD, Layibo Y, Agbétiafa K, Kolou M, Ségbéna AY. [Biermer's Disease in the Hematology Service of Campus Teaching Hospital of Lomé: 13 Cases From January 2013 to December 2014]. Mali Med. 2017;32:34-9.

4. Akinyanju OO, Okany CC. Pernicious anaemia in Africans. Clin Lab Haematol 1992; 14 : 33-44.

5. Ndiaye FSD, Fall S, Sarr A, Laraki SD, Mourtalla M, Diop TM. Données actuelles sur la maladie de Biermer. Hematologie. 2009;15:473-7.

6. Koulidiati J, Sawadogo S, Sagna Y, Somda K.S, Tieno H, Kafando E, et al. Pernicious anemia: diagnosis and course in Burkina Faso. Med Sante Trop. 2015;25:428-31.

7. Berri MA, Taous A, Boulahri T, Traibi I, Rouimi A. [Neurological Disorders Secondary to Vitamin B12 Deficiency: About 29 Cases]. Pan African Med J. 2019;32:108.

8. Berthe A, Diop MM, Diousse P, Toure PS, Faye FA, Diop BM, et al. Acquired Melanoderma: Should it be considered as a Biermer disease characteristic among black African? A 41 cases analysis. RAFMI. 2017;4:34-9.

9. Diop MM, Berthe A, Toure PS, Ndiaye FSD, Mahamat Y, Leye MY, et al. Peculiarities of Biermer disease in a senegalese internal medicine department: a 6 year prospective study. J Blood Disorders Transf. 2013;5:179.

10. Diop MM, Toure PS, Leye MY, Leye A, El Fadjri S, Diop M, et al. [Unusual aspect of pernicious anemia during association of betathalassemia: a new case report and literature review]. Mali Med. 2012;27:71-2.

11. El Euch M, Hadded S, Mahfoudhi M, Bouaziz R, Jaziri F, Ben Abdelghani $\mathrm{K}$, et al. Maladies auto-immunes au cours de la maladie de Biermer à travers une étude monocentrique. Ann Endocrinol. 2017;78:397-433.

12. Maazoun F, Frikha F, Snoussi M, Saidi N, Hriz H, Marzouk S, et al. La maladie de Biermer en milieu de médecine interne : une série de 31 cas. Rev Med Int. 2011;32S:S313-34.

13. Seynabou F, Fatou Samba Diago N, Oulimata Diop D, Abibatou Fall S, Nafissatou D. Biermer anemia: hematologic characteristics of 66 patients in a Clinical Hematology Unit at Senegal. Med Sante Trop. 2016;26:402-7.

Copyright by Julienne Teclessou, et al. This is an open-access article distributed under the terms of the Creative Commons Attribution License, which permits unrestricted use, distribution, and reproduction in any medium, provided the original author and source are credited.

Source of Support: Nil, Conflict of Interest: None declared. 